\title{
Is there any possible role for telocytes in the pathogenesis of musculoskeletal diseases?
}

\author{
Review \\ Ali Eid El Deeb', Ahmed Ali Ahmed Abd El Hafez', and Amal Ali Ahmed \\ Article \\ Abd El Hafez ${ }^{3}$ \\ Departments of ${ }^{1}$ Rheumatology Physical Medicine and Rehabilitation, ${ }^{2}$ Anesthesia And Pain \\ Management, ${ }^{3}$ Histology and Cell Biology, Faculty of Medicine, Tanta University, Egypt.
}

\begin{abstract}
Background: Telocytes (Tc) are newly discovered histological cells that are scattered within most of the tissues and organs including the musculoskeletal system. They are a peculiar type of interstitial cells with very long prolongations termed telopodes (Tp). They have also been isolated and cultured from tissues in vitro.

Some recent researches have been done handling the possible physiological function of these cells and the pathophysiological role in some musculoskeletal diseases. In any case, future functional studies of telocytes in vivo will help to understand the mechanism by which telocytes contribute to tissue homeostasis in health and disease.

Aim: We are in need to highlight on these cells to widen the scope of research about the potential hidden roles of telocytes in the pathogenesis of some rheumatic diseases.

Conclusion: Hence this review focuses on the latest progresses regarding Tc in the pathogenesis and/or repair and regeneration of different tissues like skeletal muscle, synovium and peripheral nerves.
\end{abstract}

Key Words: Musculoskeletal, system, telocyts.

Revised: 2 January 2021, Accepted: 25 May 2021.

Corresponding Author: Amal Ali Ahmed Abd El Hafez, MD, Department of Histology And Cell Biology, Faculty of Medicine, Tanta University, Egypt, Tel.: 01004517345, E-mail: Amal.bdelhafez@med.tanta.edu.eg

ISSN:2536-9172, June 2020, Vol. 4, No. 1

\subsection{Telocytes}

Telocytes (Tc) are a distinct type of interstitial cells characterized by a small cell body and extremely long and thin telopodes (Tp). The presence of Tc has been documented in many tissues and organs. Functionally, Tc form a three-dimensional (3D) interstitial network by homocellular and heterocellular communication and are involved in the maintenance of tissue homeostasis. ${ }^{[1]}$ As important interstitial cells to guide or nurse putative stem and progenitor cells in stem cell niches in a spectrum of tissues and organs, Tc contribute to tissue repair and regeneration ${ }^{[1]}$.

\subsection{Why Telocytes have a Unique, Dissimilar Cell Type?}

Tc have a small cell body with abruptly emerging very long and thin cell prolongations - telopodes (Tp). Moreover, Tp conformation is "specific", consisting of an alternation of thin fibrillar-like segments (podomers) and dilated, cistern-like regions (podoms) ${ }^{[4]}$. Podoms house mitochondria, caveolae, and components of endoplasmic reticulum (ER) the so-called calcium accumulating/ releasing units. ${ }^{[2,3]}$. Previously, their existence was either overlooked by most of the researchers, or they were confused with other interstitial cell types.

\subsection{What is the TC silhouette under electron microscope (EM)?}

TC profile has a small, oval or triangular-shaped body, and several (two to five) remarkably long, thin, and moniliform Tp. The identification of TC is mostly based on recognition of their $\mathrm{Tp}$. Tp are characterized by the following ${ }^{[4]}$

1. Number (one to five, frequently two to three).

2. Length (several tens up to hundreds of micrometers).

3. Moniliform aspect: alternation of dilated segments (podoms) and thin segments (podomers-less than $200 \mathrm{~nm}$ thickness mostly $(\sim 80 \mathrm{~nm})$, below the resolving power of light microscopy, explaining why TC were overlooked so far and why EM is mandatory for unmistakable TC identification).

4. Podoms accommodate: a) mitochondria (one or two), b) endoplasmic reticulum elements, either smooth or/ and rough ER, and c) caveolae; the so-called $\mathrm{Ca} 2+$ uptake/ release units.

5. Dichotomous branching pattern, making a 3D network, a labyrinthine system with particular intercellular 
junctions: either homocellular junctions between TC themselves or heterocellular junctions between TC and other cell types (e.g. cardiomyocytes, macrophages, stem cells etc.). ${ }^{[5]}$

\subsection{Is it not a fibroblast?}

Commonly, interstitial cells are supposed as being mainly (or even only) fibroblasts. Though it is clear that TC are not fibroblasts, some are hidden behind the hallmark "fibroblast like." Where should one draw the line(s) between the intrinsic variability of fibroblasts and their look alikes?

In fact, the so-called fibroblast-like cells were never clearly defined, the lack of specific information leading to this nomenclature. However, the dissimilarity between TC and fibroblasts is obvious as they have different ultrastructure (Table 1) and phenotype. Consequently, their functions are unlike: fibroblasts endorse collagen synthesis, while TC endorse intercellular signaling either by direct contact (junctions), or remotely (via extracellular vesicles $)^{[6]}$.

Table 1: Comparison of the Ultrastructural Characteristics of Telocytes and Fibroblasts

\begin{tabular}{lll}
\hline Feature & Fibroblasts & Telocytes \\
\hline Cell body & $\begin{array}{l}\text { Pleomorphic (phenotypic } \\
\text { heterogeneity }\end{array}$ & $\begin{array}{l}\text { Small; piriform/ } \\
\text { spindle/triangular/ } \\
\text { stellate } \\
\text { shaped; }\end{array}$ \\
Cytoplasm & Large amount & Small amount \\
Nucleus & One, oval & One, oval/rod-shaped \\
Chromatin & Typically euchromatic & Heterochromatin \\
Nucleolus & $1-2$ nucleoli & Rarely visible \\
\hline
\end{tabular}

\subsection{How do Telocytes have intercellular relationships?}

The intercellular interactions are achieved by either direct contact (intercellular junctions) or extracelluar vesicles (Evs) (extracellular vesicles are either: multivesicular bodies or shed microvesicles or exosomes). The variable Tp width might also explain the heterogeneity of the released vesicles. Thus, exosomes (with a diameter under $100 \mathrm{~nm}$ ) could be released at the podomeric level (where usual width rarely exceeds $100 \mathrm{~nm}$ ), and shed microvesicles (with a mean diameter of $180 \mathrm{~nm}$ ) could transport macromolecular signals from podoms to near cells, modifying their transcriptional activity sooner or later. The release of multi-vesicular bodies has been noted at both podomeric and podomic level. The microenvironment also plays an important role in local cellular cross talk. ${ }^{[4-7]}$

Their very long one to five cytoplasmic processes, whose width is under the resolution power of light microscopes, contrasted with their length, which is considered by some authors to be the second greatest in the human body, after that of neuronal axons, reaching up to hundreds of micrometers. The current state of knowledge indicates that telocytes form three-dimensional networks in various organs. ${ }^{[8]}$

It is known that many pathological processes originate directly from cellular changes ${ }^{[7]}$. Ten years after, telocyte announcement, several articles regarding telocytes have already been published in such prestigious journals as Nature ${ }^{[9]}$. The telocyte variety extends beyond their morphology and functions, as they have a potential role in the etiopathogenesis of different diseases. The most commonly described telocyte-associated diseases (which may be best termed "telocytopathies" in the future $)^{[8]}$. Tc relation to musculoskeletal disorders was the matter of our review.

\subsection{Skeletal muscle}

Adult skeletal muscle has a remarkable regenerative capacity after injury. The activation of the muscle stem cells, also called satellite cells (SC), is a limiting step in muscle regeneration, which enables satellite cells to proliferate, migrate and differentiate into new skeletal myocytes. Telocytes have been identified in skeletal muscle interstitium in close vicinity of blood capillaries, nerve fibres, satellite cells and myocytes, suggesting their potential roles in muscle regeneration. ${ }^{[10,11]}$ Electron microscopy showed that $\mathrm{Tp}$ were extended to neighbouring cells and interconnected by different kinds of junctions, which suggest that Tc potentially contribute to form a 3D interstitial network in skeletal muscle tissue. Shed vesicles/ exosomes released by $\mathrm{Tp}$ were also detected as found in other tissues. ${ }^{[12]}$

Because EVs have been shown to play important roles in both normal and abnormal physiology, they are also likely to be involved in the normal maintenance and degeneration of musculoskeletal tissues. Concerning the extracellular vesicles (Evs) in muscle cell differentiation, muscle regeneration, and congenital myopathies it was noted that the congenital absence of dystrophin (found in Evs) elevates the incidence of muscle injury, ultimately leading to long-term skeletal muscle dysfunction. Muscle regeneration requires the coordinate, sequential expression of various factors including secreted proteins, inflammatory cytokines, miRNAs, and membrane lipids ${ }^{[13]}$.

It is important to note here that EVs are secreted by myoblasts, while telocytes may also represent another important source of EVs in muscle. These stromal cells telopods extend into skeletal muscle interstitium. ${ }^{[4]}$

EVs can enhance muscle regeneration, and circulating muscle-derived EVs may serve as biomarkers of disease progression in congenital myopathies. Evidence from 
several in vitro studies reveals that the secretion of EVs increases during muscle differentiation contain growth factors that act as potential regulators of development, function, and repair such as basic fibroblast growth factor (bFGF), insulin-like growth factor-1 (IGF-1), transforming growth factor-beta1 (TGF-B1), and vascular endothelial growth factor (VEGF), among others (VEGF R3, PDGF) ${ }^{[14]}$. Nevertheless, these studies and those from healthy human subjects ${ }^{[15]}$ suggest that monitoring EV-derived miRNAs may serve as one approach for tracking disease progression or noninvasively assessing muscle injury following exercise. ${ }^{[16]}$

In addition to muscle satellite cells, non-satellite cells as well as many other types of cells such as bone marrowderived cells and pericytes contribute consistently to the extraordinary regenerative ability of skeletal muscle. Of note, Tc have been found in both satellite cell niche and non-satellite progenitor cell niche, forming close contacts with these two types of muscle stem cells ${ }^{[10]}$ (Fig.(Fig.1). In addition, Tc showed a peculiar migration capacity and formed a cellular network from muscle explants in vitro, which might be critical for scaffold guidance of stem/ progenitor cells in muscle regeneration. Given that Tc in skeletal muscle interstitium are VEGF and PDGFR- $\beta$ immunopositive both in situ and in vitro, Tc could also be involved in angiogenesis and vascular stability during tissue repair in skeletal muscle. ${ }^{[12]}$

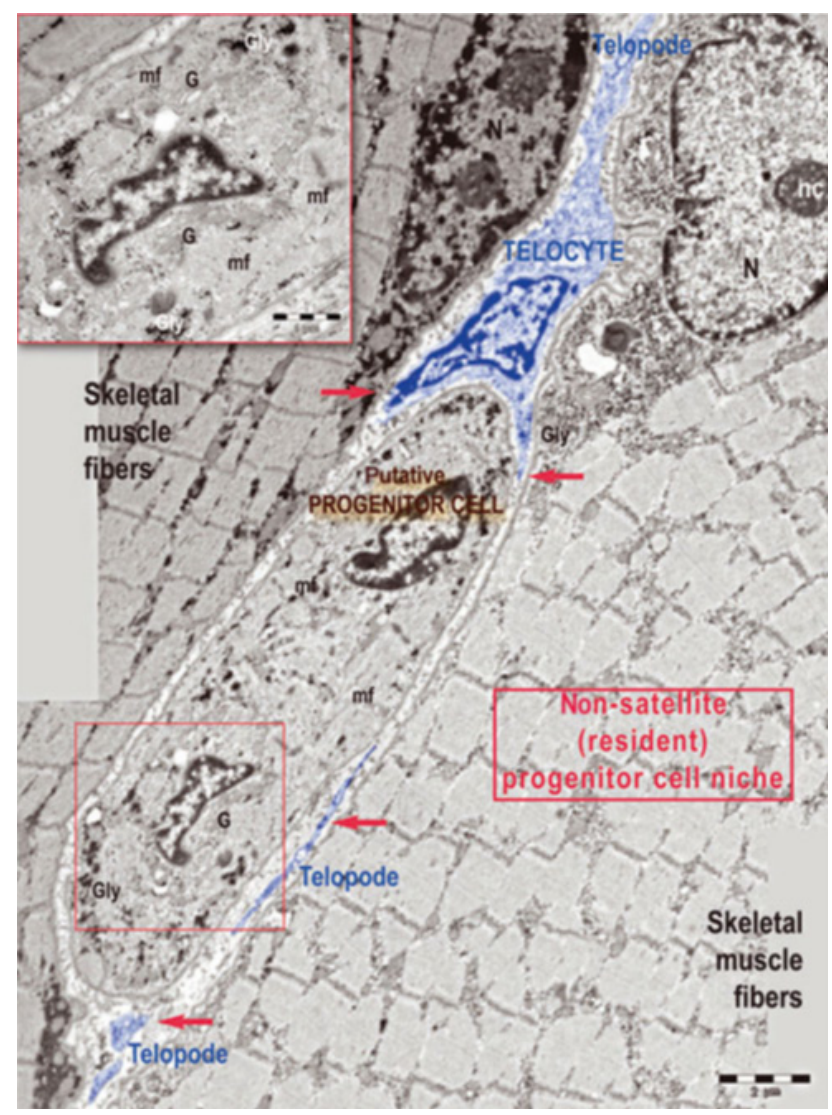

Fig. 1: A telocyte (TC) with its telopodes (Tp) around a progenitor cellin skeletal muscle. Tp are indicated with red arrows. mf: myofilaments; Gly: glycogen deposits; G: Golgi complex; $\mathrm{N}$ : nucleus; nc: nucleolus ${ }^{[12]}$
Moreover, Eccentric contraction (EC)-damaged muscle-derived Tc showed increased expression of the recognized pro-myogenic vascular endothelial growth factor-A, and SCs from the same samples exhibited increased MyoD expression denoting the role of telocytes in supporting satellite cell activation after induction of eccentric contraction- muscle injury). ${ }^{[17]}$.

\subsection{Neuromuscular spindle}

A large number of Tc were observed in NMSs. Tc formed the innermost and (partially) the outermost layers of the external NMS capsule and the entire NMS internal capsule. In the latter, the Tp were organized in a dense network, which surrounded intrafusal striated muscle cells, nerve fibres and vessels, suggesting a passive and active role in controlling NMS activity, including their participation in cell-to-cell signaling ${ }^{[18]}$. In neuromuscular spindles (NMSs), Tc may be actively involved in controlling muscle tone and motor activity ${ }^{[39]}$. Indeed, TC location is strategic and the interconnected Tc form a continuous network, which could act in cell-to-cell signaling ${ }^{[18]}$. Thus, a paracrine and/or juxtacrine intercellular modulation between Tc and myocytes has been proposed. In NMSs, homo- and heterocellular communications of Tc could therefore occur by means of shed vesicles and close apposition as has been suggested for Tc and myocytes. ${ }^{[18]}$

\subsection{Synovium}

In this context, studies provide the evidence that Tc are part of the microscopic anatomical structure of synovium, a thin connective tissue membrane that covers intraarticular surfaces of the fibrous joint capsule and serves as an important source of nutrients and lubricants for diarthrodial joints. Indeed the probable functions of synovial Tc according to the different roles that have been ascribed to the $\mathrm{Tc}$ in other organs ${ }^{[1]}$ the three-dimensional network formed by telopodes within the sublining layer might be gifted with mechanical support, intercellular communication and regulatory properties making a substantial contribution to the maintenance of synovial homeostasis. Noteworthy, the location of numerous Tc and telopodes at the boundary between the synovial lining and sublining layers and in close vicinity of blood microvessels supports this assumption. ${ }^{[17]}$

Tc in the structure of human synovium, lays the foundations for upcoming studies on synovial Tc roles in a variety of degenerative and destructive joint diseases. using dynamic methods either in vitro or in vivo may discover the possible functions of synovial Tc the might indeed provide new clues for their potential therapeutic applications in joint regenerative medicine. ${ }^{[17]}$ 

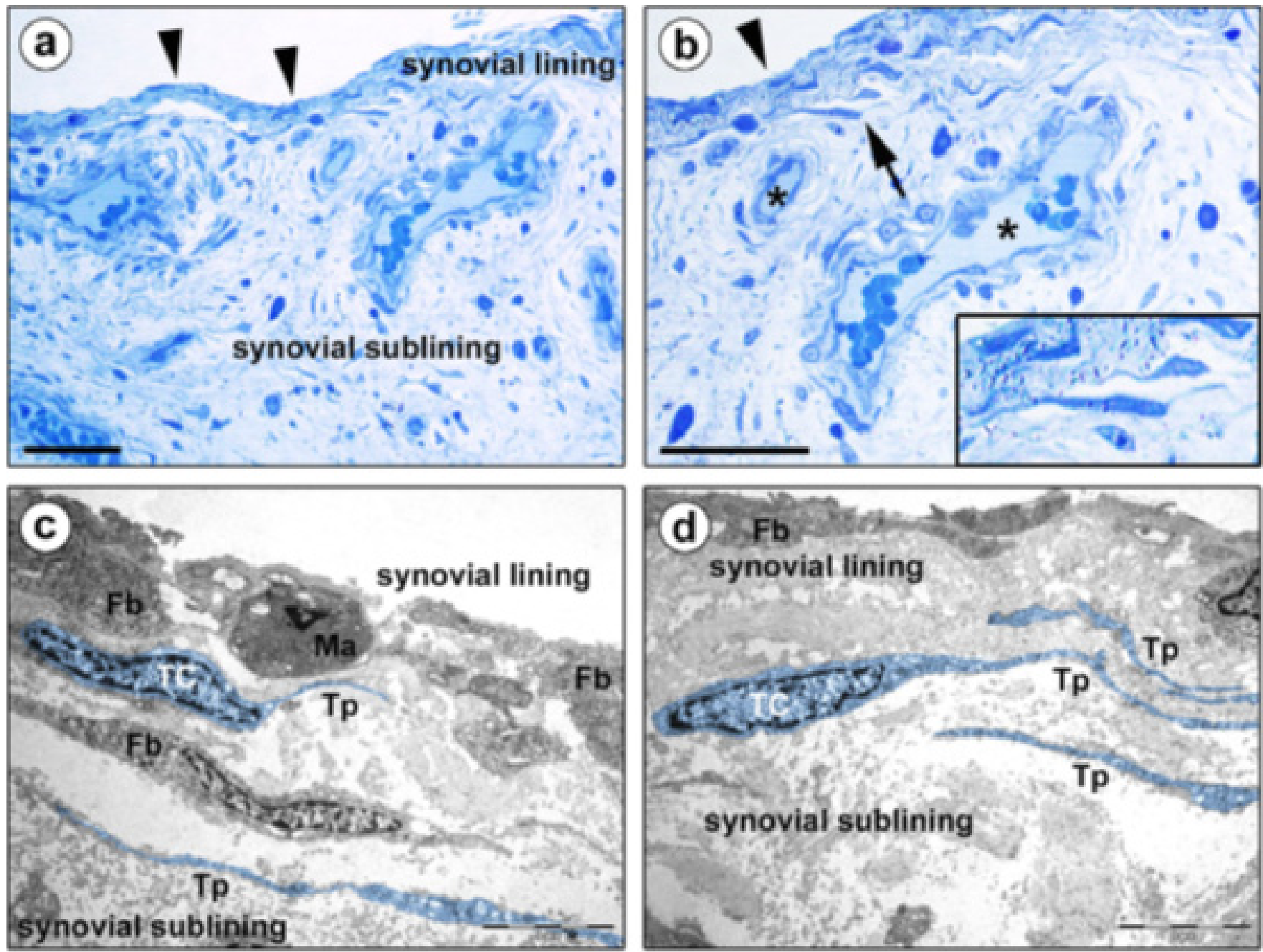

Fig. 2: Morphology of human synovium (lining and sublining layers) under light and transmission electron microscopy. (a,b) Representative photomicrographs of toluidine blue-stained synovial semithin sections.

Arrowheads in $(a, b)$ point to the synovial lining layer, while asterisks in (b) denote blood vessels in the synovial sublining layer. (b) Stromal cells exhibiting a spindle-shaped or piriform cell body with a large nucleus and very small amount of cytoplasm, and long and thin cytoplasmic processes are observed at the boundary between the synovial lining and sublining layers (arrow; shown at higher magnification in the inset). (c,d) Representative transmission electron microscopy photomicrographs of synovial ultrathin sections stained with uranyl acetate and bismuth subnitrate solutions. (TC) and (Tp) have been digitally colored in blue. The ultrastructural traits of telocytes are: i) a spindleshaped or piriform cell body with a relatively large euchromatic nucleus surrounded by a thin cytoplasmic layer containing few mitochondria, scarce cisternae of endoplasmic reticulum and a small Golgi apparatus, and ii) the presence of telopodes, long cytoplasmic processes with a narrow emergence from the cell body and a moniliform silhouette characterized by the alternation of thin segments (podomers) and expanded parts (podoms). Telocytes and telopodes are present in the synovial sublining layer immediately beneath the type A/macrophagelike synoviocytes $(\mathrm{Ma})$ and the type $\mathrm{B} /$ fibroblastlike synoviocytes $(\mathrm{Fb})$ constituting the synovial lining $(\mathrm{c}, \mathrm{d})$. The synovial sublining contains either telopodebearing telocytes or fibroblasts $(\mathrm{Fb})$, these latter displaying an abundant cytoplasm rich in cisternae of rough endoplasmic reticulum, mitochondria and Golgi apparatus, and short and thick processes (c). Scale bar: $50 \mu \mathrm{m}(\mathrm{a}, \mathrm{b}), 5 \mu \mathrm{m}(\mathrm{c}, \mathrm{d}){ }^{\left[{ }^{[17]}\right.}$

\subsection{Peripheral Nervous System}

Telocytes/CD34+ stromal cells were studied in the normal and pathological peripheral nervous system (PNS) ${ }^{[19]}$. The following aspects were considered: (A) general characteristics of telocytes and the terminology used for these cells (e.g., endoneurial stromal cells) in PNS; (B) the presence, characteristics and arrangement of telocytes in the normal PNS, including (i) nerve epiperineurium and endoneurium (e.g., telopodes extending into the endoneurial space); (ii) sensory nerve endings (e.g., Meissner and Pacinian corpuscles, and neuromuscular spindles); (iii) ganglia; and (iv) the intestinal autonomic nervous system; (C) the telocytes in the pathologic PNS, encompassing (i) hyperplastic neurogenic processes (neurogenic hyperplasia), highly demonstrative of telocyte characteristics and relations, (ii) PNS tumours, such as neurofibroma, schwannoma, granular cell tumour and nerve sheath myxoma, and interstitial cell of Cajalrelated gastrointestinal stromal tumour (GIST), (iii) tumour-invaded nerves and (iv) traumatic, metabolic, degenerative or genetic neuropathies, in which there 
are fewer studies on telocytes, e.g., neuroinflammation and nerves in undescended testicles (cryptorchidism), Klinefelter syndrome, crush injury, mucopolysaccharidosis II (Hunter's syndrome) and Charcot-Marie-Tooth disease. ${ }^{[19]}$

\subsection{Telocytes extracellular vesicles in rheumatoid arthritis and osteoarthritis:}

The chronic inflammation of the joints that accompanies rheumatoid arthritis RA), and the mechanical degradation of articular cartilage that accompanies osteoarthritis (OA), each appear to involve changes in the EVs circulating within the joint space. In the case of RA, EVs can present antigens to activate the innate and adaptive immune system, leading to inflammation within the joint, and the EVs also transport inflammatory cytokines such as TNF-and IL-1 ${ }^{[20]}$.

Extracellular matrix (ECM) maintenance is very important for joint health and there is a balance between synthesis and degradation of ECM; when this balance is altered, the patient is at risk for the pathology seen in $\mathrm{OA}^{[20]}$. The two main important cells related to these processes are chondrocytes, which help synthesize the extracellular matrix of articular cartilage and fibroblastlike synoviocytes (FLS), which secrete synovial fluid. EVs are involved in communication between these different cell types. For example, EVs isolated from chondrocytes treated with IL-1bcan directly increase MMP-13 production in FLS cells ${ }^{[20]}$. miRNAs carried by EVs are also implicated in the development of OA. When FLS cells are treated with Il-1b,EVs are secreted that show elevated levels of miR-500B, miR-4454, miR-720, miR-199b, and miR-3154. [21, 22], [16]

\section{CONCLUSION}

In summary, we have systemically reviewed the most recent studies of the potential significance of Tc in tissue repair and regeneration in skeletal muscle, synovium, peripheral nerves, RA, OA. However, it has to be noted that up till now, the studies of Tc were mainly based on the morphology aspect, while direct evidence to show the roles of Tc in diseases and regenerative medicine is still in need for more exploration. In such circumstances, it is necessary to explore TC-specific markers useful to identify the presence of Tc in a more specific and easier way in tissues. In addition, the co-culture of Tc with other cells and the use of $3 \mathrm{D}$ culture environment and tissue engineering in vitro, to further confirm and clarify the mechanisms by which Tc contribute to tissue pathogenesis, repair and regeneration. Also, a deeper understanding of the relationship between Tc and stem/progenitor cells is of great need. All these studies will be useful to clarify the biological functions of Tc in tissue repair and regeneration, as well as to provide new insights into the potential therapeutic values of Tc in muscloskeleta diseases.
By targeting Tc alone or in tandem with stem cells, we might promote pharmacological or non-pharmacological methods to enhance the growth of Tc would be a novel therapeutic strategy besides exogenous transplantation for many diseased disorders.

\section{CONFLICT OF INTEREST}

There are no conflicts of interest.

\section{REFERENCES}

1. Cretoiu, D., et al., Telocytes heterogeneity: From cellular morphology to functional evidence. Semin Cell Dev Biol, 2017. 64: p. 26-39.

2. Suciu, L., M.I. Nicolescu, and L.M. Popescu, Cardiac telocytes: serial dynamic images in cell culture. Journal of Cellular and Molecular Medicine, 2010. 14(11): p. 2687-2692.

3. Kim, J.E., et al., Single cell and genetic analyses reveal conserved populations and signaling mechanisms of gastrointestinal stromal niches. Nat Commun, 2020. 11(1): p. 334

4. Popescu, The tandem: telocytes - stem cells. Int J Biol Biomed Eng 2011 5: p. 83-92.

5. $\mathrm{Xu}, \mathrm{Y}$., et al., Immunohistochemical biomarkers and distribution of telocytes in ApoE(-/-) mice. Cell Biol Int, 2019. 43(11): p. 1286-1295.

6. Cretoiu, S.M. and L.M. Popescu, Telocytes revisited. Biomol Concepts, 2014. 5(5): p. 353-69.

7. Varga, I., et al., Recently Discovered Interstitial Cell Population of Telocytes: Distinguishing Facts from Fiction Regarding Their Role in the Pathogenesis of Diverse Diseases Called "Telocytopathies". Medicina (Kaunas), 2019. 55(2).

8. Varga, I., et al., Recently discovered interstitial cells termed telocytes: distinguishing cell-biological and histological facts from fictions. Biologia, 2018. 74(2): p. $195-203$.

9. Shoshkes-Carmel, M., et al., Author Correction: Subepithelial telocytes are an important source of Wnts that supports intestinal crypts. Nature, 2018. 560(7718): p. E29.

10. L. M .Popescu, et al., Identification of telocytes in skeletal muscle interstitium: implication for muscle regeneration. Journal of cellular and molecular medicine, 2011. 15(6): p. 1379-1392. 
11. Bojin, F.M., et al., Telocytes within human skeletal muscle stem cell niche. Journal of cellular and molecular medicine, 2011. 15(10): p. 2269-2272.

12. Bei, Y., et al., Telocytes in regenerative medicine. J Cell Mol Med, 2015. 19(7): p. 1441-54.

13. Wang, H. and B. Wang, Extracellular vesicle microRNAs mediate skeletal muscle myogenesis and disease (Review). Biomed Rep, 2016. 5(3): p. 296300 .

14. Do, M.-K.Q., et al., Time-coordinated prevalence of extracellular HGF, FGF2 and TGF- $\beta 3$ in crush-injured skeletal muscle. Animal Science Journal, 2012. 83(10): p. 712-717.

15. Guescini, M., et al., Muscle Releases AlphaSarcoglycan Positive Extracellular Vesicles Carrying miRNAs in the Bloodstream. PLOS ONE, 2015. 10(5): p. e0125094.

16. Murphy, C., et al., Emerging role of extracellular vesicles in musculoskeletal diseases. Mol Aspects Med, 2018. 60: p. 123-128.
17. Rosa, I., et al., Morphological evidence of telocytes in human synovium. Sci Rep, 2018. 8(1): p. 3581.

18. Diaz-Flores, L., et al., Telocytes in neuromuscular spindles. J Cell Mol Med, 2013. 17(4): p. 457-65.

19. Diaz-Flores, L., et al., Telocytes in the Normal and Pathological Peripheral Nervous System. Int J Mol Sci, 2020. 21(12).

20. Withrow, J., et al., Extracellular vesicles in the pathogenesis of rheumatoid arthritis and osteoarthritis. Arthritis research \& therapy, 2016. 18(1): p. 286-286.

21. Kato T Fau - Miyaki, S., et al., Exosomes from IL-1 stimulated synovial fibroblasts induce osteoarthritic changes in articular chondrocytes. (1478-6362 (Electronic)).

22. Kato, T., et al., Exosomes from IL-1 $\beta$ stimulated synovial fibroblasts induce osteoarthritic changes in articular chondrocytes. Arthritis research \& therapy, 2014. 16(4): p. R163-R163. 


\section{الملخص العربى}

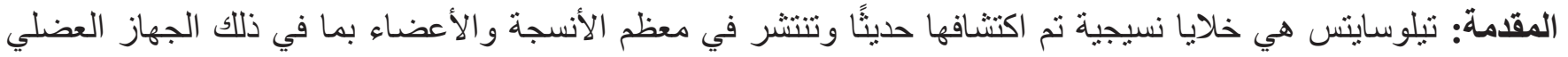

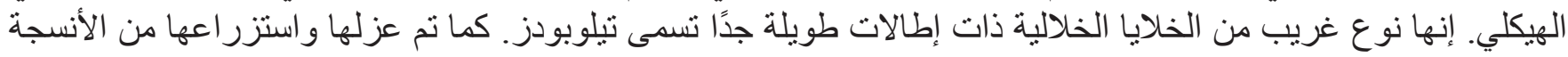
في المختبر.

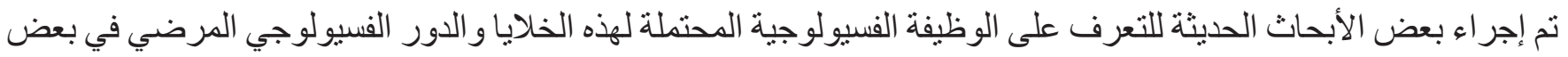

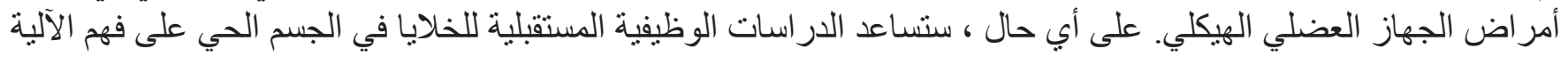

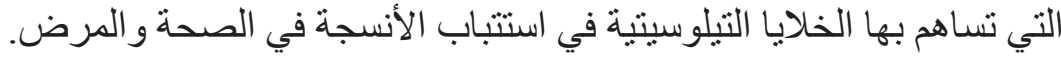

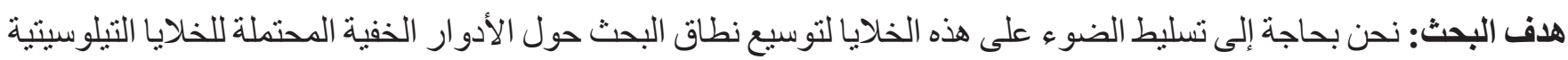

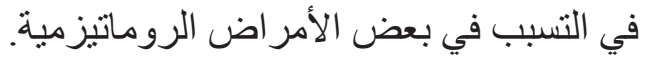

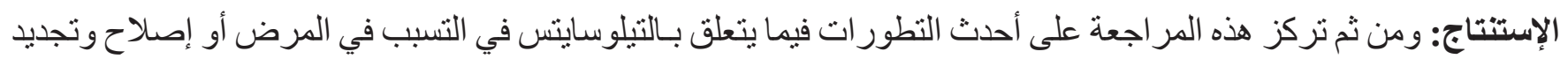
الأنسجة المختلفة مثل العضلات الهيكلية و الغشاء الزيلية اليلي و الأعصاب الطرفية. 